\title{
YASSIR BENHIMA
}

\section{Quelques aspects de \\ l'historiographie des transferts techniques en Méditerranée médiévale}

Le fait technique demeure sans doute le parent pauvre d'une histoire de la Méditerranée médiévale qui s'est développée, ces dernières décennies, d'une manière exponentielle. Les raisons d'une telle désaffection des chercheurs sont nombreuses: elles relèvent tant de considérations méthodologiques et documentaires que des effets de la construction et de l'articulation des champs disciplinaires.

Cette étude porte sur les seules réalités techniques et ne couvre pas deux autres domaines traditionnellement associés à l'histoire des techniques: l'histoire de la culture matérielle et l'histoire des sciences. C'est ainsi qu'il semble nécessaire de distinguer d'emblée les techniques de production de l'ensemble des composantes de la culture matérielle. Celle-ci, selon la formulation aboutie et critique qu'en donne Jean-Marie Pesez, couvre tous les rapports entre l'homme et la matière, depuis l'acquisition jusqu'à la consommation, en passant par les conditions, les moyens et les processus de transformation ${ }^{1}$.

Si les techniques de production se distinguent clairement de la culture matérielle, leur étude historique est souvent associée aux recherches sur l'histoire des sciences. Cette confusion peut se justifier par la continuité et la perméabilité des différentes formes de la connaissance humaine, mais elle demeure néanmoins problématique pour deux raisons. C'est que la place réservée au fait technique dans son ensemble reflète d'abord une hiérarchisation ancienne des savoirs, héritée de la culture médiévale (aussi bien dans l'Occident chrétien que dans le monde musulman): cela relève plutôt d'un processus de distinction sociale, basé sur la valorisation du travail intellectuel et déconsidérant la technique et le travail manuel et matériel. La confusion entre les domaines technique et scientifique est également due aux difficultés inhérentes à la recherche sur le premier. Le monde des techniques de production échappe le plus souvent à la sphère de l'écrit; leur connaissance n'est possible que par le biais d'une littérature hétérogène qui impose des filtres culturels entre le réel et l'écrit ${ }^{2}$.

1 Selon cette définition, les éléments de la culture matérielle sont: »1) les moyens de travail (l'homme, les outils); 2) l'objet du travail (les richesses matérielles, les matières premières);

3) l'expérience de l'homme dans le processus de production (les techniques); 4) l'utilisation des produits matériels (la consommation)«. Jean-Marie PESEZ, Archéologie du village et de la maison rurale au Moyen Âge, Lyon 1998 (Collection d'histoire et d'archéologie médiévale, 5), p. 22.

2 Philippe Braunstein, Travail et entreprise au Moyen âge, Bruxelles 2003 (Bibliothèque du Moyen Âge, 21), p. 23-24. 
En envisageant le fait technique dans ses dimensions sociales et économiques, l'histoire des techniques ne peut faire l'économie d'une confrontation systématique des textes (de toute nature: normatifs, narratifs, actes de la pratique s'il en existe), des données archéologiques et des résultats de l'archéométrie (analyse des vestiges archéologiques par les méthodes des sciences exactes, notamment la physique et la chimie). Et, comme toute approche située au carrefour de plusieurs disciplines, ce parti méthodologique se heurte à la contingence habituelle des savoirs académiques et à leur construction.

Cette spécificité de l'objet de l'histoire des techniques a forcément conditionné la conception du fait technique dans les sociétés de la Méditerranée médiévale, et par conséquent l'histoire des circulations et des transferts techniques. Dans ce contexte, aborder le rôle et la place des techniques de l'Islam méditerranéen se heurte ainsi à un ensemble de présupposés théoriques et méthodologiques qu'il convient de déconstruire dans un premier temps, avant de montrer l'importance des acquis, mais aussi les limites de la recherche sur le sujet. On reviendra ensuite sur la nécessité d'une meilleure contextualisation des faits techniques en transfert dans le pourtour méditerranéen.

\section{TENDANCES ET CARACTÉRISTIQUES DES RECHERCHES SUR L'HISTOIRE DES TRANSFERTS TECHNIQUES EN MÉDITERRANÉE MÉDIÉVALE}

L'histoire des techniques a longtemps été envisagée dans le cadre d'une vision globale et transpériodique. Le fait technique, par ses temporalités différentielles (ballotté entre un temps long des permanences et le caractère souvent événementiel de l'acte d'invention tel que le conçoit la société industrielle moderne), se prêterait à des approches sur la longue durée. Cette tendance expliquerait d'ailleurs la multiplication des entreprises individuelles ou collectives de ce genre. Des sondages dans les ouvrages les plus représentatifs de cette catégorie permettent de faire quelques remarques.

Dans l'»Histoire générale des techniques« dirigé par Maurice Daumas - ouvrage un peu daté maintenant - un chapitre d'une trentaine de pages est consacré aux techniques du monde musulman. Si la qualité scientifique de son auteur, Gaston Wiet, alors professeur au Collège de France, ne fait aucun doute, son texte témoigne du caractère encore embryonnaire de la recherche sur les structures techniques et économiques anciennes du monde musulman ${ }^{3}$. L'ambiguïté des notions utilisées pour qualifier l'objet du chapitre dénote un flottement dans les concepts utilisés: l'auteur évoque dans son introduction les difficultés d'écrire une histoire des mœurs qui intègre les différentes facettes matérielles du vécu quotidien, en associant, de la sorte, l'étude des procédés techniques à celle des manifestations de la culture matérielle. Ainsi, quand il traite des »techniques de la vie citadine«, il décrit surtout les principales composantes

3 Gaston WIET, Le monde musulman, dans: Maurice DAUMAS (dir.), Histoire générale des techniques. Tome I: Les origines de la civilisation technique, Paris 1962, p. 339-373, ici p. 339 . 
de la ville islamique et son organisation urbaine. Dans un autre passage sur la construction, la technique de la țābiya (pisé), longuement décrite par Ibn Haldūn, est confondue avec la fabrication du mortier, pouvant faire appel aux mêmes ingrédients mais pour un usage bien différent ${ }^{4}$. Dans ce tableau général brossé à grands traits, on comprend la part infime accordée aux phénomènes de transferts et de diffusion. Ainsi, dans le point dévolu au papier et aux textiles, s'il est fait état de la transmission de la technique de la fabrication du papier depuis la Chine vers le monde musulman, son transfert vers l'Occident par le biais de l'Andalus et de la Sicile n'est point évoqué. On pourrait multiplier les exemples des lacunes et des limites de cet essai; il demeure néanmoins une contribution sérieuse et surtout le reflet, avant tout, d'un état des connaissances sur les techniques du monde musulman au terme de la première moitié $\mathrm{du} \mathrm{XX}^{\mathrm{e}}$ siècle.

Sans vouloir exagérer les limites et les failles inhérentes à toute contribution synthétique, il me semble intéressant de souligner quelques topoï souvent reproduits par ce type de publications. »L'histoire des techniques « dirigé par Bertrand Gille, spécialiste incontesté de l'histoire des techniques en France, en est un autre exemple. Quelques remarques peuvent être formulées sur le passage concernant les techniques du monde musulman médiéval et leurs liens avec d'autres aires culturelles. La première relève de la catégorie dans laquelle fut classé le point sur les techniques du monde musulman: ainsi après un chapitre sur »les grandes civilisations techniques« (consacré à l'Antiquité orientale du Néolithique jusqu'aux Phéniciens), et un autre sur les systèmes grec et romain (puis byzantin, considéré comme l'héritier direct de Rome), un chapitre fourre-tout intitulé "systèmes bloqués« regroupe les parties consacrées au monde musulman, à la Chine et à l'Amérique précolombienne. Ce chapitre, curieusement inséré avant celui dévolu au Moyen Âge occidental, est en quelque sorte intemporel. Il concerne, selon la logique de l'auteur, des systèmes clos, incapables de se renouveler, laissés en marge de l'histoire. Pour l'auteur, ces systèmes techniques traditionnels bloquent toute mutation interne et ne sont pas cohérents avec les autres systèmes économique, social et scientifique 5 . Cette situation est encore plus problématique pour les techniques du monde musulman, car non seulement le système est bloqué, mais il est l'héritier de systèmes antérieurs aussi moins lotis, notamment du système grec.

Au-delà du caractère inopportun de la notion de »blocage«, il est frappant que cette contribution véhicule surtout une conception téléologique et unilinéaire de l'évolution des systèmes techniques. Ce blocage présumé, érigé en caractéristique structurelle d'un système donné, n'est qu'une projection d'une situation contemporaine marquée par la domination de la technologie occidentale. Il ne rend pas compte des évolutions variables, et n'explique pas non plus les périodes de crise qu'a connues l'assise technique de la production économique, agricole ou industrielle, en terre d'islam. Il traduit enfin

4 Ibid., p. 347-348. Sur les techniques de construction en terre, voir Mohammed HAMMAM (dir.), L'architecture de terre en Méditerranée, Rabat 1999 (Publications de la faculté des lettres et des sciences humaines - Rabat, Colloques et séminaires, 80).

5 Bertrand Gille, Histoire des techniques, Paris 1978 (Encyclopédie de la Pléiade, 41), p. 466. 
une contingence artificielle des systèmes techniques intégrant peu la place des transferts techniques (dans tous les sens, vers et depuis le mode musulman).

Cette vision qu'offre Gille souffre aussi, et cela n'est pas applicable à la seule histoire des techniques, d'un essentialisme ravageur. Concevoir le monde musulman comme un espace unitaire et uniforme, en dépit de traditions techniques très diversifiées, participe de la persistance de ce type de cliché. Une étude récente sur les techniques antiques a d'ailleurs relevé le même constat: parler des techniques de l'Empire romain sans prendre en considération l'hétérogénéité de ses provinces relève du même genre de généralisations ${ }^{6}$. Cet essentialisme politico-culturel s'accompagne souvent, d'ailleurs, même dans des publications très récentes, de caractérisations ethniques. Les Grecs étaient plus des théoriciens que des praticiens de la technologie. Les Romains sont plus doués d'un grand sens de la pratique 7 . Quant au monde musulman, les synthèses, comme celle de Gille, ne retiennent que le mode de vie nomade des populations arabes, jugé - selon une lecture évolutionniste de l'histoire -, responsable d'une très grande incompétence technique.

Cette vision plutôt négative est vite dissipée quand on examine les études plus spécifiquement consacrées à l'Islam médiéval. Ici, le système technique musulman est vu comme réceptacle des traditions antérieures mais aussi comme foyer de diffusion vers l'Occident latin. Si l'on ne peut rendre compte de tout ce qui a pu être écrit à cet égard, une mention particulière doit être faite de certains travaux phares. En représentant emblématique de l'esprit des Annales pour l'histoire de la Méditerranée du haut Moyen Âge, Maurice Lombard, mort prématurément en 1965, avait entamé une série d'enquêtes sectorielles dans lesquelles le fait technique était envisagé sous l'angle d'une histoire sociale et économique qui se voulait encore `totale`, embrassant de la sorte des espaces très larges. Ses deux ouvrages posthumes, consacrés respectivement aux métaux et aux textiles, s'appuyant sur des matériaux abondants et une cartographie novatrice pour l'époque, illustrent bien sa préoccupation de fournir une synthèse circonstanciée des connaissances sur les deux thèmes tout en revisitant, par leur biais, la thèse de Pirenne8.

Dans un effort comparable à Lombard, quoique d'une manière moins systématique, les travaux d'Eliyahu Ashtor sur l'histoire économique du Proche-Orient réservent une place non négligeable au fait technique pour expliquer le déclin économique de l'Orient à la fin du Moyen Âge. Dans plusieurs de ses articles, comme celui consacré à »l'ascendant technologique de l'Occident«, il passe en revue les différentes thèses

6 Kevin GReEne, Technology and Innovation in Context: the Roman Background to Mediaeval and Later Developments, dans: Journal of Roman Archaeology 7 (1994), p. 22-33, spécialement p. 24-25.

7 Ibid., p. 25.

8 Maurice LOMBARD, Les métaux dans l'ancien monde du $\mathrm{V}^{\mathrm{e}}$ au XI $\mathrm{XI}^{\mathrm{e}}$ siècle, Paris 1974 (Civilisations et sociétés, 38); ID., Les textiles dans le monde musulman du $\mathrm{VII}^{\mathrm{e}}$ au XII ${ }^{\mathrm{e}}$ siècle, Paris 1978. Ces études d'ensemble complètent une série d'articles sur l'histoire économique de la Méditerranée au haut Moyen Âge, dans lesquels l'auteur montre une sensibilité aux techniques et aux circulations matérielles (l'or, le bois et les produits de chasse notamment). Voir ID., Espaces et réseaux du haut Moyen Âge, Paris 1972 (Le savoir historique, 2). 
proposées pour expliquer la stagnation des procédés techniques, voire la déperdition d'acquis techniques dans différents domaines: textiles, savonnerie et verrerie9.

Si l'histoire économique a constitué un terreau fertile pour le développement de l'histoire des techniques, l'histoire des sciences a également longtemps alimenté nos connaissances sur les techniques de précision et en général sur les applications pratiques de la culture scientifique théorique. Les travaux de Donald Hill, notamment son ouvrage en collaboration avec Ahmad al-Hassan, témoignent de cette approche ${ }^{10}$. Cet ouvrage accessible et richement documenté, qui constitue la principale synthèse sur l'histoire des techniques dans le monde musulman médiéval, passe en revue les différents domaines de la production technique, en mettant en valeur les manifestations d'innovations et l'apport de l'aire arabo-musulmane à la sphère technique en général. Dans la même lignée, soulignons l'intérêt notable pour les techniques du monde musulman et pour leurs transferts dans une encyclopédie anglo-saxonne récente associant également les techniques à l'histoire des sciences ${ }^{11}$.

Si l'on ne peut complètement dissocier le développement technique des connaissances scientifiques théoriques, il est indubitable que l'association croissante des deux domaines est une conséquence de la culture technique contemporaine. La projection d'une telle vision sur les réalités médiévales, quand le fait technique était surtout le fruit d'un savoir empirique, peut être problématique. En effet, cette tradition académique se nourrit aussi des différences sémantiques que couvrent les termes »technique« et »technologie«. Si le premier est le plus utilisé dans un contexte francophone, le second prédomine dans la littérature scientifique anglo-saxone. Leurs définitions réciproques reflètent des conceptions différentes de l'objet étudié. Ainsi le terme »technique« définit toute action socialisée sur la matière, et donc les procédés utilisés pour transformer une matière première en objet (artefact). La technologie s'intéresse essentiellement au savoir nécessaire pour la mise en œuvre des procédés techniques ${ }^{12}$.

Aux différences des notions utilisées correspondent des perspectives contrastées à l'égard de l'histoire du fait technique. Si la première, dans le cadre d'une démarche plutôt descriptive, s'attache à rendre compte des procédés techniques utilisés dans différents domaines, la seconde s'emploie principalement à l'identification des processus d'évolution du savoir technique. Le développement de la pensée technique, ses

9 Eliyahu ASHTOR, Technology, Industry and Trade. The Levant Versus Europe, 1250-1500, Variorum, Londres 1992 (Collected studies series, 372).

10 Ahmad Y. AL-Hassan, Donald R. HiLl, Sciences et techniques en Islam, Paris, 1991. C'est dans ce cadre que l'on pourrait aussi classer le point sur la technologie que Hill rédigea pour Roshdi RASHED (dir.), Histoire des sciences arabes. 3: technologie, alchimie et sciences de la vie, Paris 1997, p. 11-54.

11 Thomas Glick, Steven J. Livesey, Faith Wallis (dir.), Medieval Science, Technology, and Medicine. An Encyclopedia, New York, Londres 2005.

12 Robert Cresswell, Technologie, dans: Pierre BOnTE, Michel IZARD (dir.), Dictionnaire de l'ethnologie et de l'anthropologie, Paris 2000, p. 698-701; Willeke WeNDRICH, Body Knowledge. Ethnoarchaeological Learning and the Interpretation of Ancient Technology, dans: Bernard Mathieu, Dimitri MeEKS, Myriam Wissa (dir.), L'apport de l'Égypte à l'histoire des techniques. Méthode, chronologie et comparaisons, Le Caire 2006 (Bibliothèque d'étude/ Institut français d'archéologie orientale, 142), p. 267-275. 
assises scientifiques et son cadre sociopolitique et économique, sont essentiellement appréhendés dans cette dernière perspective selon leurs apports innovants.

\section{APERÇU DES PRINCIPAUX THÈMES DE LA RECHERCHE SUR LES TECHNIQUES DE L'ISLAM MÉDITERRANÉEN}

L'évolution de l'histoire des techniques dans l'Islam méditerranéen et ses liens avec l'Occident latin a suivi l'éclatement, l'émiettement de la pratique historienne. Les essais synthétiques de Lombard n'ont pas été réactualisés, mais un développement notable des études sectorielles peut être souligné. Aussi, l'étude des techniques médiévales et de leur circulation fait appel d'une manière croissante aux services de l'archéologie, de l'archéométrie voire de l'ethnoarchéologie. Ces disciplines viennent compléter, par une approche centrée sur les artefacts et les procédés techniques, les données textuelles qui alimentent les études des historiens de l'économie et des sciences. Elles pallient très souvent l'absence totale de témoignages écrits: les progrès considérables réalisés dans le domaine de la connaissance des techniques de l'Afrique subsaharienne, dans la métallurgie ou dans les textiles, en constituent un parfait exemple $^{13}$.

S'il n'est pas question de procéder à un fastidieux inventaire des thèmes étudiés, on peut néanmoins citer les principaux champs de recherche. On peut ainsi noter, de prime abord, l'importance des travaux sur deux domaines souvent interdépendants: l'hydraulique et les techniques agricoles, connues notamment grâce au développement considérable de l'archéologie. Le cas d'al-Andalus est emblématique de cet intérêt, non seulement en raison de la dynamique de la recherche archéologique sur la Péninsule en général, mais aussi en raison d'un contexte historique particulièrement propice à l'étude des situations de transferts culturels. L'accent a été mis dans ces travaux sur l'utilisation des ressources hydrauliques, leur inscription dans un système socioéconomique et politique basé sur la prédominance de la petite propriété foncière. La mise en valeur des terroirs irrigués d'al-Andalus a été possible grâce à ce qu'on a pris le parti d'appeler la petite hydraulique - une hydraulique essentiellement paysanne. Le transfert de cette dernière à la société chrétienne coloniale au lendemain de la Reconquête a entrainé, non pas une rupture au niveau des procédés techniques, mais plutôt une transformation des modes de la gestion sociale et économique des moyens

13 Voir par exemple Dominique CARDON, Pour une archéologie des textiles en Afrique occidentale et au Maghreb: genèse de l'outillage textile et étude des colorants, dans: André BAZZANA, Hamady Bocoum (dir.), Du nord au sud du Sahara. Cinquante ans d'archéologie française en Afrique de l'Ouest et au Maghreb, Paris 2004, p. 307-314, et dans le même volume Paul BENOîT, Elisée Coulibaly, Philippe Fluzin, Le laboratoire, lieu de coopération, p. 325-330. Sur la production de l'or, voir Jean DEVISSE, L'or, dans: ID. (dir.), Vallées du Niger, Paris 1993, p. 344-357. 
techniques de la production, désormais conditionnée par les normes sociales et le système foncier de la société conquérante ${ }^{14}$.

Parallèlement au développement des techniques hydrauliques, la place accordée à l'acclimatation, la généralisation de l'exploitation de plusieurs espèces d'origine tropicale, puis leur diffusion dans l'ensemble du bassin méditerranéen, est représentative de ce qu'on a appelé la »révolution agricole« de l'Islam médiéval. La seule synthèse disponible sur le sujet - celle d'Andrew Watson - est malheureusement datée. Nos connaissances sur ce sujet peuvent évoluer sensiblement grâce à l'exploitation des traités agronomiques, mais aussi en raison de l'apport indispensable de l'archéobotanique ${ }^{15}$.

Avec les techniques hydrauliques, les arts du feu constituent un champ capital de la recherche sur les transferts techniques en Méditerranée médiévale. Sous cette appellation, on regroupe traditionnellement trois domaines différents par l'ampleur de leurs productions et formes d'utilisations (céramique, verrerie, métallurgie), mais qui émanent de sphères techniques perméables (surtout entre céramique et verre). L'étude des arts du feu a connu, ces dernières décennies, une révolution majeure en raison du développement de l'archéométrie, qui vient appuyer, par les résultats des analyses chimico-physiques en laboratoire, les approches formelles ou fonctionnalistes plutôt conventionnelles.

L'étude des techniques céramiques, sans doute la plus évoluée pour l'aire islamoméditerranéenne, a permis de mettre au jour des formes de transfert qui étaient jusquelà restées à l'ombre de l'érudition classique. Quelques cas emblématiques illustrent les avancées d'une recherche exponentielle ${ }^{16}$, comme les céramiques en vert et brun ou à lustre métallique ${ }^{17}$. La production du verre, qui gagne encore à être mieux connue dans la partie occidentale de l'Islam méditerranéen, est concernée par le développement des

14 Parmi les centaines d'études sur l'hydraulique d'al-Andalus, on peut citer quelques unes des plus représentatives: Thomas GLICK, Regadío y sociedad en la Valencia medieval, Valence 1988; Miquel Barcelo, Helena KirChNer, Carmen NaVARro, El agua que no duerme. Fundamentos de la arqueología hidráulica andalusí, Grenade 1996; Patrice CrESSIER (dir.), La maîtrise de l'eau en al-Andalus. Paysages, pratiques et techniques, Madrid 2006 (Collection de la Casa de Velázquez, 93).

15 Andrew M. WATSON, Agricultural Innovation in the Early Islamic World. The Diffusion of Crops and Farming Techniques (700-1100), Cambridge 1983. L'ouvrage récent de Mohamed OUERFELLI, Le sucre. Production, commercialisation et usages dans la Méditerranée médiévale, Leyde 2008 (The medieval Mediterranean, 71), ne se limite pas à l'étude de la diffusion de la canne à sucre, mais élargit son travail aux différents usages culinaires et pharmacologiques du sucre et aux réseaux marchands qui en ont assuré la commercialisation. Sur les traités agronomiques andalous et leur exploitation, voir le récent bilan bibliographique de Expiración GarCiA, Julia María CARABAZA, Studies on the Agronomy of al-Andalus, dans: Revue des mondes musulmans et de la Méditerranée 126 (2009), http://remmm.revues.org/index 6465.html [15/12/2009] (15/3/2011).

16 Comme en témoigne la masse considérable d'informations archéologiques et archéométriques sur les céramiques médiévales, telle qu'elle apparaît par exemple dans les actes des congrès de l'AIECM2 (Association internationale de l'étude de la céramique médiévale en Méditerranée).

17 Voir par exemple Le vert et le brun, de Kairouan à Avignon, céramiques du $\mathrm{X}^{\mathrm{e}}$ au $\mathrm{XV}^{\mathrm{e}}$ siècle, catalogue d'exposition, Paris 1995; Jeannette Rose-Albrecht (dir.), Le calife, le prince et le potier. Les faïences à reflets métalliques, Paris 2002. 
recherches, qui ont noté notamment le rôle des techniques proche-orientales dans l'évolution des productions italiennes ${ }^{18}$.

Pour la métallurgie la situation est plus mitigée car, à l'exception du domaine iranien ${ }^{19}$, les techniques métallurgiques dans la Méditerranée musulmane restent encore mal connues ${ }^{20}$. Les rares études ponctuelles révèlent la place capitale dans le sujet qu'occupent les questions de transfert et d'évolution des techniques, en considérant non seulement les deux rives de la Méditerranée, mais également la riche tradition de l'Afrique subsaharienne - tradition dont la connaissance a par ailleurs considérablement évolué ces trois dernières décennies.

L'ingénierie et la technologie de précision ont suscité aussi l'intérêt des chercheurs. Les travaux de Donald Hill constituent à cet égard de nombreux exemples de l'application des techniques arabes dans le domaine des machines et des mécanismes. Les enquêtes sur ce thème reposent sur les traités arabes médiévaux consacrés au sujet: elles reflètent le développement de la technologie mécanique et la maîtrise de techniques introduisant un contrôle automatique du mouvement ${ }^{21}$. C'est dans ce sens que Donald Hill suggère l'origine arabo-islamique des principes techniques qui ont présidé à l'apparition de l'horloge mécanique en Occident22.

L'évolution de la production du papier et ses liens avec le développement des usages de l'écrit en Méditerranée mérite la multiplication des enquêtes systématiques. Si plusieurs recherches retracent le double transfert du papier, depuis la Chine vers l'Empire abbasside d'un côté, puis de l'extrême Occident musulman vers le monde latin, en Sicile et en Espagne d'un autre côté, plusieurs aspects de ses techniques de fabrication et les modalités de sa diffusion sont à étudier. Parmi les questions qui se posent à ce propos, on peut noter les raisons et les conditions de la lenteur du transfert du papier arabe vers l'Europe, puis la dynamique que prit rapidement sa fabrication en Europe au point d'inonder le marché maghrébin à la fin du Moyen Âge23.

18 Voir par exemple Catherine Hess (dir.), The Arts of Fire. Islamic Influences on Glass and Ceramics of the Italian Renaissance, Los Angeles 2004.

19 Notamment James W. Allan, Persian Metal Technology 700-1300 AD, Londres 1979 (Oxford Oriental Institute monographs, 2).

20 Voir LOMBARD, Les métaux dans l'ancien monde (voir n. 8); James W. AlLAN, The Influence of the Metalwork of the Arab Mediterranean on that of Medieval Europe, dans: Dionisius AgiUs, Richard HitchCOCK, The Arab Influence in Medieval Europe, Reading 1994 (Middle East Cultures series, 18), p. 44-62; Alberto CANTO, Patrice CREssier (dir.), Minas y metalurgia en al-Andalus y Magreb occidental. Explotación y poblamiento, Madrid 2008 (Collection de la Casa de Velázquez, 102).

21 Donald HiLl, A History of Engineering in Classical and Medieval Times, Londres 1984.

22 Ibid., p. 142. Sur ce point, voir également Jean GIMPEL, La révolution industrielle au Moyen Âge, Paris 1975, p. 141-160.

23 Sur le transfert du papier de l'Occident musulman vers l'Occident latin, voir notamment Robert BuRns, The Paper Revolution in Europe: Crusader Valencia's Paper Industry, dans: Pacific Historical Review 50 (1981), p. 1-30 et Pierre GUICHARD, Du parchemin au papier, dans: Comprendre le XIII' siècle, Lyon 1995, p. 161-172. Sur l'histoire du papier dans le monde musulman, de son transfert depuis la Chine jusqu'à la Méditerranée, Jonathan BLOOM, Paper Before Print. The History and the Impact of Paper in the Islamic World, New Haven 2001; Gene- 
Depuis les travaux pionniers de Robert Serjeant et de Maurice Lombard24, la connaissance des techniques du textile dans le monde arabo-mulsuman a beaucoup progressé. Au-delà des approches esthétiques et économiques des textiles et de leur circulation, le développement de l'archéologie des textiles et des études techniques des textiles anciens a joué un rôle fondamental à ce propos ${ }^{25}$. Même si les études d'ensemble se font rares $^{26}$, on considère que le transfert de plusieurs techniques ou éléments d'outillage du monde musulman est à l'origine de développements dans les techniques de la draperie occidentale ${ }^{27}$. De même, la production du cuir, concernant aussi bien les objets destinés à l'habillement que des artefacts divers pour la conservation et la préparation alimentaire, pour la reliure, ou encore l'armement et la cavalerie, reste très peu connue. Ainsi les historiens ne se sont pas intéressés à l'industrie du cuir au Maghreb, qui fournissait abondement le marché européen, ni à son éventuel impact sur les techniques occidentales ${ }^{28}$.

Le travail du bois, matériau considéré comme un produit stratégique en terre d'islam, demeure également dans l'ombre. On connaît peu, par exemple, les techniques de construction navale en Méditerranée en comparaison avec l'océan Indien ${ }^{29}$. Les usages civils du bois, notamment dans la construction, sont inégalement étudiés. Le cas d'al-Andalus reste le plus représentatif, notamment pour la charpenterie dans les époques tardives ${ }^{30}$.

Enfin, le domaine de l'armement et des techniques militaires constitue l'un des champs de recherche les plus féconds dans l'identification des transferts techniques possibles entre Islam et monde latin. La plupart des recherches sur ce thème concer-

viève HUMBERT, Le manuscrit arabe et ses papiers, dans: Revue des mondes musulmans et de la Méditerranée 99-100 (2002), p. 55-77.

24 Robert SERJEANT, Islamic Textiles: Material for a History up to the Mongol Conquest, Beyrouth 1972; LOMBARD, Les textiles (voir n. 8).

25 On peut souligner à cet égard l'apport capital d'une entreprise comme le CIETA (Centre international d'étude des textiles anciens).

26 On peut citer comme exemple représentatif l'étude de Dominique CARDON, La draperie au Moyen Âge. Essor d'une grande industrie européenne, Paris 1999.

${ }_{27}$ Ce serait le cas par exemple du métier à tisser à chaîne horizontale. Sur le débat suscité par cette question, ibid., p. 391-394 et 400-412.

28 Parmi les rares exceptions à cet égard, soulignons le travail de Ricardo CORDOBA DE LA LlaVE (dir.), Mil años del trabajo del cuero: actas del II simposio de historia de las técnicas, Cordoue 2003.

${ }_{29}$ Voir à ce propos le travail de Christophe PICARD, L'océan Atlantique musulman. De la conquête arabe à l'époque almohade. Navigation et mise en valeur des côtes d'al-Andalus et du Maghreb occidental (Portugal, Espagne, Maroc), Paris 1997, p. 291-321. En guise de comparaison avec le golfe arabo-persique et l'océan Indien, Dionisius AGIUS, Classic Ships of Islam, Leyde 2008.

30 C'est le cas de l'excellente étude de Carmen LÓPEZ PERTIÑEZ, La carpintería en la arquitectura nazarí, Grenade 2006. Sur la place des artisans mudéjars dans le transfert des techniques d'alAndalus vers l'Espagne chrétienne puis vers l'Amérique, voir Enrique NuERE, La carpintería de armar castellana. Su influjo islámico y exportación al nuevo mundo, dans: Santiago SEbastián LóPez, Ignacio Henares CuÉllar, Alfredo J. Morales, El mudéjar iberoamericano. Del islam al nuevo mundo, Grenade 1995, p. 51-58. 
nent la période des croisades ${ }^{31}$, alors que ces questions sont paradoxalement peu étudiées pour l'Occident musulman ${ }^{32}$. Le développement des études comparatives est amené à approfondir notre connaissance de l'évolution parallèle des techniques militaires en Orient et en Occident.

La liste des domaines techniques encore à explorer est longue. Cet aperçu, ne prétendant à aucune exhaustivité, n'en fournit que des exemples, censés attester les acquis de la recherche et certaines de ses lacunes.

\section{LA CONTEXTUALISATION DES TRANSFERTS TECHNIQUES EN MÉDITERRANÉE MÉDIÉVALE}

Dans l'usage contemporain, la notion de transfert technique est désormais associée au processus de diffusion des équipements, des techniques ou des savoir-faire depuis les pays développés vers le tiers monde ${ }^{33}$. Elle caractérise de la sorte une relation asymétrique et induit une dépendance du récepteur à l'égard du transmetteur. Cette vision restrictive de la notion de transfert technique induit forcément l'établissement d'une hiérarchisation des systèmes techniques, et privilégie la lecture des rapports entre cultures en termes de dettes de l'une vers l'autre.

De cette optique découle la réduction de l'histoire des transferts techniques à des listes commentées d'innovations, ou en l'absence d'éléments clairs à ce propos, des premières attestations d'utilisations d'un objet déterminé. La mise en liaison des différentes mentions est très souvent interprétée en termes de diffusion. Des travaux importants, comme ceux de Lynn White Jr. ${ }^{34}$, ou encore d'Andrew Watson, sont profondément fondés sur une lecture diffusionniste. Sous l'influence de l'archéologie du $\mathrm{XIX}^{\mathrm{e}}$ siècle ou du courant diffusionniste de l'anthropologie culturelle britannique et

31 Les travaux de David Nicolle sont sans doute les plus connus sur ces transferts. Voir son recueil d'articles David NiCOLle, Warriors and Their Weapons Around the Time of the Crusades, Aldershot 2002 (Collected studies series, 756); ID. (dir.), Companion to Medieval Arms and Armour, Woolbridge 2002, en particulier la troisième partie de l'ouvrage (p. 105-221). Sur les techniques militaires et la conduite de la guerre, Abbès ZouACHE, Armées et combats en Syrie (491/1098-569/1174). Analyse comparée des chroniques médiévales latines et arabes, Damas 2008 (PIFD, 230). Voir aussi [mars 2009] http://ifpo.revues.org/222 (24/11/2011). Pour une période plus ancienne voir Robert HoYLAND, Brian GiLmour, Medieval Islamic Swords and Swordmaking. Kindi's Treatise on Swords and Their Kinds (edition, translation, and commentary), Exeter 2006.

32 Pour la péninsule Ibérique, aussi bien du côté musulman que chrétien, voir Alvaro SOLER DEL CAMPO, La evolución del armamento medieval en el reino castellano-leonés y al-Andalus, Madrid 1993.

33 Kevin GreEne, Historiography and Theoretical Approaches, dans: John Peter Oleson (dir.), The Oxford Handbook of Engineering and Technology in the Classical World, Oxford 2008, p. 79.

34 Lynn White Jr., Technologie médiévale et transformations sociales, Paris 1969 (Civilisations et sociétés, 13). 
américaine ${ }^{35}$, l'accent est mis sur les circulations, la dispersion, l'imitation ou encore l'emprunt d'artefacts et de savoir-faire; autant de notions qui ne favorisent guère une réelle contextualisation des faits étudiés.

Or, la contextualisation d'un transfert technique s'impose, comme d'ailleurs pour toutes les autres formes de transferts culturels. Une technique transférée ne peut être dissociée du milieu technique qui a vu son développement, ni de son contexte général (social, politique, économique...). Elle ne trouve son sens que si elle est appréhendée dans un ensemble technique (appelé »système « ou »milieu« en fonction des auteurs) et culturel cohérent ${ }^{36}$. Étudier les transferts techniques reviendrait donc à traiter d'une technique, de son arrière-plan technologique et de ses implications socioculturelles et économiques, aussi bien avant qu'après sa transmission et sa diffusion, dans les aires culturelles concernées. Ainsi, en guise d'exemple, le transfert de la technique de la fabrication du papier de l'Occident musulman à l'Occident latin, en Italie et en Espagne, s'inscrit dans le cadre d'un processus complexe. Il intervient après que le papier de fabrication arabe eut été largement utilisé, à un moment où l'expansion des pratiques notariales crée une demande considérable pour de nouveaux supports de l'écrit. La circulation de la main-d'œuvre qualifiée, et surtout son passage sous la domination chrétienne, comme dans le cas de la ville de Šătiba, conquise en 1242, facilite l'appropriation de la technique dans l'espace aragonais. Le développement des techniques de fabrication en Occident et l'affaiblissement des capacités de production au Maghreb rend propice la prépondérance de la production européenne dans l'approvisionnement du marché maghrébin, fait connu grâce à une fatwā célèbre du $\mathrm{XV}^{\mathrm{e}}$ siècle $^{37}$. Dans cette histoire où s'imbriquent données techniques, usages sociaux, juridiques et culturels, sans oublier l'impact de la conjoncture politique et économique, le transfert technique devient anecdotique s'il est envisagé en dehors des évolutions respectives des sociétés concernées.

La nécessité de contextualisation passe également par une réflexion sur les échelles de l'analyse historique. En embrassant des aires culturelles immenses (Occident, Islam, Asie...) composées de sous-ensembles régionaux très hétérogènes, les approches classiques et généralistes de l'histoire des techniques tombent dans le piège inéluctable

35 Brita RuPP-EISENREICH, Diffusionnisme, dans: BONTE, IZARD (dir.), Dictionnaire de l'ethnologie (voir n. 12), p. 201.

36 Sur la notion de »système technique«, voir l'élaboration théorique de GILLE, Histoire des techniques (voir n. 5) p. 10-28; André LeroI-Gourhan, Milieu et technique, Paris 1992 ( $1^{\text {re }}$ éd. 1945), p. 340-343, avait défini les critères élémentaires d'un milieu technique, caractérisé notamment par sa perméabilité et sa continuité dans le temps et l'espace. La principale étude d'ensemble d'un système technique en terre d'islam reste celle de Parviz MoHEBBI, Techniques et ressources en Iran du $\mathrm{VII}^{\mathrm{e}}$ au $\mathrm{XIX}^{\mathrm{e}}$ siècle, Téhéran 1996 (Bibliothèque iranienne, 46).

37 Cette fatwā d'Ibn Marzūq (1364-1438), compilée dans al-Wanšarīsī, al-Mi'yār al-mu'rib wa alğāmi' al-muğrib 'an fatāwā 'ulamā' Ifrīqiya wa'l-Andalus wa 'l-Mag̉rib, Rabat 1981, t. 1, p. 75 107, est citée dans plusieurs études. Elle a récemment fait l'objet d'un examen détaillé par Leor HALEVI, Christian Impurity versus Economic Necessity: A Fifteenth-Century Fatwa on European Paper, dans: Speculum 83 (2008), p. 917-945. Pour l'histoire du transfert du papier en Méditerranée médiévale, voir les références citées dans la note 23. 
de l'essentialisme et des généralisations abusives. Pourtant, affiner la lecture des transferts techniques imposerait un examen multidimensionnel et multiscalaire qui permettait, par le biais de la variation des échelles de l'analyse, d'appréhender les niveaux différents de l'interaction entre le technique et le socioculturel, mais aussi de restituer l'impact différentiel d'une technique donnée au niveau local ou régional ${ }^{38}$. C'est seulement au prix d'une approche appropriée que la valorisation des manifestations de convergence, de métissage ou d'hybridation technique permettrait de dépasser les écueils du culturalisme ${ }^{39}$.

L'effort de contextualisation d'un transfert technique doit aussi aboutir à une réflexion sur la place du procédé concerné dans la culture technique, aussi bien de la société d'origine que celle d'accueil. Cela permet de placer l'innovation technique, en tant que développement d'un élément inventé pour une utilisation pratique ou commerciale et son adaptation au contexte socioculturel, dans un ensemble technique complexe. Dans ce dernier, des manifestations de traditions ou la persistance de formes anciennes et la continuité de leur utilisation ne traduisent pas forcément une stagnation technique, mais la reproduction de solutions qui ont prouvé leur efficacité. Centrer la réflexion sur la nouveauté et l'innovation n'est-il pas un avatar d'une survalorisation de l'invention comme seul critère d'évolution (ou de modernité) technique?

L'étude des transferts techniques en Méditerranée médiévale peut trouver dans le comparatisme quelques pistes intéressantes. Tout en évitant la comparaison comme source de classification des cultures dans le cadre d'une lecture évolutionniste des faits techniques, ce comparatisme peut nourrir une réflexion sur les situations de nondiffusion ou de refus. Autant qu'un transfert identifié, le refus participe clairement de la définition et de la caractérisation d'un système socioculturel. Un tel constat a été maintes fois évoqué, notamment dans des travaux désormais classiques de Fernand Braudel, qui considère le refus d'emprunter, selon sa terminologie, comme un élément discriminant de toute société. Pour lui, »certains de ces refus s'accompagnent même d'une conscience, d'une lucidité aiguë, si d'autres sont aveugles, comme déterminés par des seuils ou des verrous qui interdisent les passages « ${ }^{40}$.

Se pencher sur les raisons de tels refus permet d'éviter les apories de lectures simplistes, présentant une sélection d'ordre socioculturel comme un obstacle structurel. Des études récentes peuvent servir d'exemple, tel un article d'Andrew Watson sur un cas de non-diffusion, celui de la non-adoption du système de l'open field dans l'agriculture d'al-Andalus. La démonstration de l'auteur a surtout insisté sur l'inadéquation de ce mode d'organisation de l'espace agraire conçu pour une économie agropastorale régie par le système féodal, avec les conditions socio-économiques en alAndalus où prédominait une agriculture de jardinage dont les formes d'association

38 Sur l'importance et la portée de la variation de l'échelle de l'analyse historique dans l'étude des techniques médiévales, voir Liliane PÉREZ, Catherine VERNA, La circulation des savoirs techniques du Moyen Âge à l'époque moderne. Nouvelles approches et enjeux méthodologiques, dans: Tracés. Revue de sciences humaines 16 (2009), p. 25-61, surtout p. 40-45.

39 Ibid., p. 50-51.

40 Fernand BRAUDEL, Écrits sur l'histoire, Paris 1969, p. 293. 
avec l'élevage intensif ne sont guère évoquées par les sources ${ }^{41}$. Dans un tout autre domaine, la question de la lenteur de la diffusion de l'imprimerie dans le monde ottoman pourrait être expliquée, non pas par des considérations techniques ou économiques, mais surtout par la puissance des corporations de copistes qui officiaient dans les différentes métropoles de l'Empire, et dont la survie en tant que catégorie socioprofessionnelle s'accompagnait d'une hostilité à l'égard de la nouvelle invention ${ }^{42}$.

En s'interrogeant sur les raisons d'un refus, la recherche en histoire des techniques peut dépasser les écueils d'une lecture qui focalise exclusivement sur les phénomènes de diffusion comme principal moyen de progrès et d'évolution technique. Le cas de l'Afrique, considérée comme un continent techniquement défaillant et victime d'un retard structurel, montre le risque que constitue la projection de réalités contemporaines sur la lecture des périodes anciennes, selon une vision téléologique de l'histoire ${ }^{43}$.

L'étude des techniques en général, et de leur processus de transfert en particulier, doit prendre en considération les limites et les distorsions qu'engendre une lecture selon le seul prisme de la littérature savante. Car l'on est souvent confronté à la question du caractère expérimental de certains procédés techniques attestés par une documentation savante, et le degré de leur étendue ou de leur généralisation. Cette interrogation a été longtemps posée à propos des traités d'agronomie andalous par exemple, dont l'extrême richesse et le caractère savant pourraient laisser dubitatif quant à l'ampleur de la diffusion des techniques attestées.

L'attention de l'historien des faits sociaux et économiques pourrait ainsi porter sur la nécessité d'établir une stratigraphie des savoirs techniques. Cette archéologie du savoir technique pourrait répondre à plusieurs objectifs. D'abord, fournir un tableau représentant le plus fidèlement possible la diversité des savoirs et savoir-faire techniques dans un domaine donné, sans insister sur les seules innovations. On doit également essayer de déceler les formes d'articulation entre, d'un côté, les savoirs constitués, construits sur une base scientifique et répondant aux normes d'une société lettrée, et, d'un autre un milieu d'acteurs praticiens dont le savoir-faire est acquis au terme d'une transmission essentiellement orale et empirique. Le postulat du cloisonnement du monde des techniques, en opposition au caractère ouvert de la pensée scientifique, mérite d'être

41 Andrew M. WAtson, A Case of Non-Diffusion: The Non-Adoption by Muslim Spain of the Open-field System of Christian Europe. Causes and Consequences, dans: Simonetta CAVACIOCCHI (dir.), Relazioni economiche tra Europa e mondo islamico secc. XIII-XVIII, Florence 2007 (Atti delle settimane di studi et altri convegni, 38), p. 241-264.

42 William ClaRENCE-SMITH, Scientific and Technological Interchanges between the Islamic World and Europe ca. 1450-ca. 1800, dans: CAVACIOCCHI (dir.), Relazioni economiche (voir n. 41), p. 719-737, en particulier p. 725. Francis RoBINSON, Technology and Religious Change: Islam and the Impact of Print, dans: Modern Asian Studies 27/1 (1993), p. 229-251. Sur la réception d'autres innovations techniques dans l'Empire ottoman, Rudolph PETERS, Religious Attitudes Towards Modernization in the Ottoman Empire. A Nineteenth Century Pious Text on Steamships, Factories and the Telegraph, dans: Die Welt des Islams 26 (1986), p. $76-$ 105.

43 Pour un aperçu général des raisons historiques et écologiques des spécificités du système technique africain, voir Ralph A. AUSTEN, Daniel HEADRICK, The Role of Technology in the African Past, dans: African Studies Review 26/4 (1983), p. 163-184. 
revu en faveur d'une valorisation des différents modes de transmission et de communication aussi bien langagière que sensorielle ${ }^{44}$.

Enfin, il convient de réfléchir sur la terminologie associée aux différentes techniques en tant que reflet d'un système de classification et d'identification et d'un mode d'organisation du travail. La taxinomie des techniques, référant souvent à des origines géographiques réelles ou fictives, témoigne aussi des systèmes de représentation et d'identification et des circulations marchandes ${ }^{45}$.

\section{CONCLUSION}

La réflexion sur les tendances de l'histoire des transferts techniques en Méditerranée médiévale se heurte avant tout à un grand éclatement du savoir. La rareté des synthèses et la multiplicité des études sectorielles accentuent la difficulté d'échafauder une vision cohérente des différents systèmes techniques et de situer les faits techniques par rapport aux évolutions sociales, politiques, économiques et culturelles de l'espace méditerranéen. En revanche, la richesse des matériaux disponibles grâce au concours de sources et d'approches diverses permet d'entrevoir des perspectives prometteuses, et surtout de soumettre les faits étudiés à des questionnements en adéquation avec la complexité et la superposition des traditions techniques des différentes parties de l'espace méditerranéen.

44 Sur le caractère ouvert de la connaissance technique, PÉREZ, VERNA, La circulation des savoirs techniques (voir n. 38), p. 29-30. Sur les formes, autres que l'écrit, de transmission des savoirs, voir par exemple WENDRICH, Body Knowledge (voir n. 12); Arianna BORRELLI, Aspects of the Astrolabe. architectonica ratio in Tenth- and Eleventh-Century Europe, Stuttgart 2008 (Sudhoffs Archiv, cah. 57).

45 Sur ce point, PÉREZ, VernA, La circulation des savoirs techniques (voir n. 38), p. 34-35. 